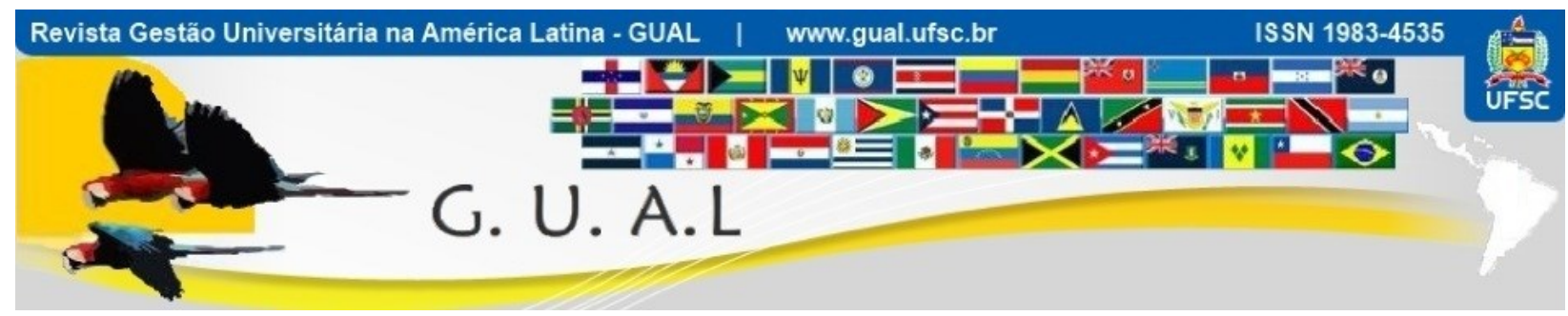

DOI: http://dx.doi.org/10.5007/1983-4535.2017v10n1p280

\title{
IMERSÃO INTERNACIONAL: UMA AVALIAÇÃO DE INTERCAMBISTAS DE MESTRADO PROFISSIONAL
}

\section{INTERNATIONAL IMMERSION: AN EVALUATION BY EXCHANGE STUDENTS FROM PROFESSIONAL MASTER}

Alexandre Nascimento Almeida, Doutor

Universidade de Brasília - UnB alexalmeida@unb.br

André Nunes, Doutor Universidade de Brasília - UnB andrenunes@unb.br

Andréa de Oliveira Gonçalves, Doutora

Universidade de Brasília - UnB andreagon@unb.br

Luiz Honorato da Silva Junior, Doutor Universidade de Brasília - UnB lula honorato@hotmail.com

Recebido em 27/agosto/2015

Aprovado em 19/outubro/2016

Sistema de Avaliação: Double Blind Review

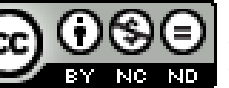

Esta obra está sob uma Licença Creative Commons Atribuição-Uso. 


\title{
RESUMO
}

A internacionalização de cursos das universidades envolve o fluxo de pessoas, informações e, eventualmente, recursos que se movem, tanto na direção das instituições estrangeiras com as quais se mantêm laços de cooperação acadêmica, quanto na direção oposta. Esse processo pode acarretar vantagens acadêmicas pela melhoria da formação, do ensino e da pesquisa e necessita ser continuamente avaliado a fim de aperfeiçoar os seus benefícios. Nesse sentido, este trabalho apresenta uma avaliação da iniciativa de imersão internacional de um mestrado profissional conforme a percepção do discente. Após 15 dias de atividades em Portugal, 26 alunos responderam um questionário com perguntas relacionadas à efetividade da imersão. Em geral, os resultados indicaram uma contribuição extremamente importante para a maioria das atividades realizadas, sugerindo uma relação de benefício/custo muito favorável da atividade. A principal dificuldade apontada pelos discentes para participação na imersão esteve relacionada à limitação e burocracia para obtenção de recursos em suas instituições de trabalho.

Palavras-chave: Internacionalização. Universidade. Avaliação discente.

\begin{abstract}
The internationalization of university courses involves the flow of people, information and possibly resources that move in the direction of foreign institutions with which it maintains academic cooperation ties, as in the opposite direction. This process can lead to academic advantages by improving training, education and research in the institution, and needs to be continuously assessed in order to improve its benefits. Therefore, this work presents an evaluation of international immersion initiative of a professional master's degree according the perception of the student. After 15 days of activities in Portugal, 26 students answered a questionnaire related to the effectiveness of the immersion program. In general, the results indicated a contribution extremely important for most activities, pointing to a benefit/cost relation favorable of the activity. The main difficulty pointed out by students for participation in the immersion was related to restrictions and bureaucracy to obtain resources in their work institutions.
\end{abstract}

Keywords: Internationalization. University. Student evaluation. 


\section{INTRODUÇÃO}

A internacionalização trata de tema complexo e muitos são os documentos que abordam suas concepções teóricas. E no pensamento de Morosini (2006), a internacionalização da educação superior é um conceito complexo, com uma diversidade de termos relacionados, apresentando diversas fases de desenvolvimento. São citadas: a) dimensão internacional - presente no século XX, que se caracteriza por ser uma fase incidental mais do que organizada; b) educação internacional - atividade organizada prevalente nos Estados Unidos, entre a segunda guerra mundial e o término da guerra fria, preferentemente por razões políticas e de segurança nacional; e c) internacionalização da educação superior, posterior à guerra fria e com características de um processo estratégico ligado à globalização e à regionalização das sociedades e seu impacto na educação superior.

Na definição de Bartell (2003) citado por Morosini (2006) a internacionalização é vista como trocas internacionais relacionadas à educação, bem como é possível realizá-la com a presença de estrangeiros e estudantes-convênios num determinado campus; concessões de pesquisa internacional; projetos de pesquisa internacionais cooperativados; associações internacionais envolvendo consultoria para universidades estrangeiras e outras instituições; setores de universidades privadas com metas internacionais; cooperação internacional e colaboração entre escolas, conselhos e faculdades na universidade; e o grau de imersão internacional no currículo e muitas outras formas.

Em meio aos conceitos apresentados, o Programa de Pós-graduação Stricto Sensu em Gestão Pública (PPGP) da Universidade de Brasília propõe em seu projeto pedagógico o cumprimento de ações de internacionalização segundo as diretrizes da CAPES. Essas ações visam o aperfeiçoamento e trocas de natureza acadêmica e profissional entre discentes e docentes. No primeiro semestre de 2015, discentes matriculados no curso de mestrado profissional do PPGP participaram de uma atividade de imersão no Instituto Superior de Ciências Sociais e Políticas (ISCSP) da Universidade de Lisboa, em Portugal. A adesão na atividade contou com 26 alunos que foram acompanhados por dois professores do PPGP e pela vice-diretora da Faculdade UnB de Planaltina - FUP.

Os alunos do PPGP participaram de aulas do mestrado em Administração Pública do ISCSP e visitaram diversos órgãos relacionados com a gestão pública de Portugal, totalizando cinco visitas técnicas. 
Tendo em vista a busca de informação para melhorar a atividade de imersão realizada, a mesma foi avaliada pelos alunos participantes. Especificamente, os objetivos buscaram avaliar a percepção dos alunos em relação aos seguintes aspectos:

- Contribuição da imersão para a sua formação

- O custo/benefício da imersão para a sua instituição de origem

- O grau de dificuldade enfrentado para a participação na imersão

\section{FUNDAMENTOS TEÓRICOS}

Desde meados dos anos 1980, a dimensão internacional vem se tornando central na agenda das Instituições de Ensino Superior - IES, das organizações estudantis e das agências de certificação acadêmica, tornando prioridade para os governos em suas políticas para educação (LAUS, 2012). Como resultado, vários estudos foram publicados sobre as políticas e estratégias de internacionalização, dando origem a uma nova área de pesquisa em educação superior como, por exemplo, os trabalhos de Rudzki (1998), Knight (1994), Davies (2001) e Knight (2004).

Segundo Sebastián (2004), a internacionalização da universidade é um instrumento para melhoria de sua qualidade e tem um caráter dual, dado que transforma a estrutura interna, mas também a projeção externa da instituição. Na mesma linha, para Kinght (2004) a dimensão internacional do ensino superior está se tornando cada vez mais importante, complexa e confusa, elevando a importância de estudos que abordem o tema.

A internacionalização não acarreta apenas vantagens institucionais (importância e renome) ou acadêmicas (melhoria da formação do ensino e da pesquisa). No Brasil, ela assume relevância adicional para as IES, uma vez que a Coordenação de Aperfeiçoamento de Pessoal de Nível Superior - CAPES a toma como condição para a concessão das notas máximas de avaliação no âmbito da pós-graduação, principalmente para programas de doutorado. A obtenção dessas notas, por sua vez, amplia o reconhecimento das IES e do programa bem avaliado, além de lhes permitir o acesso a certos recursos financeiros, tais como os provenientes do Programa de Excelência Acadêmica (PROEX), exclusivos para programas com reconhecido nível de excelência.

Porém, a avaliação da efetividade da internacionalização de um programa de pósgraduação ainda carece de aprimoramento, não estando pacificadas as divergências em torno dos critérios de avaliação da CAPES. Para Marrara (2007), devem-se discutir quais os 
indicadores capazes de comprovar se as políticas de internacionalização de determinada IES se coadunam com os objetivos extraídos da Constituição, da Lei de Diretrizes e Bases da Educação - LDB e do Plano Nacional de Pós-Graduação - PNPG, bem como, em quais dados esses indicadores devem-se basear.

Não existe um consenso sobre a valoração de estratégias de internacionalização, algumas táticas como, por exemplo: oferta de cursos internacionalmente populares, organização de eventos de porte internacional, atração de alunos e pesquisadores estrangeiros, incentivo de publicações dos pesquisadores em periódicos internacionais, possuem restrições nas visões de Denman (2004), Kok (2005) e Mok (2006). Para esses autores, essas estratégias residem na ideia de internacionalização, mais como uma ferramenta de marketing a favor da promoção do nome da IES e da busca de novos consumidores para seus serviços, motivada geralmente por interesses financeiros, do que pelo ânimo de colaborar como o desenvolvimento científico e educacional.

Portanto, em primeiro lugar, a avaliação da internacionalização deveria considerar como as estratégias adotadas beneficiam seu corpo discente e docente no exercício de suas atividades acadêmicas internas (art. 214 e 218 da CR); em segundo lugar, dever-se-ia verificar em que medida esses benefícios se revertem em favor da solução de problemas nacionais ou comuns da humanidade (art. 4 da CR combinado com o art. 43 da LDB e com as diretrizes do PNPG) (MARRARA, 2007).

\section{MATERIAL E MÉTODOS}

\subsection{ATIVIDADES REALIZADAS NA IMERSÃO}

Os alunos do PPGP participaram de 26 horas aula no mestrado em Administração Pública do ISCSP e visitaram cinco órgãos relacionados com a gestão pública de Portugal (Tabela 1). Cada visita técnica teve a duração de 4 horas/aula, totalizando 20 horas aula. O detalhamento da agenda e algumas fotos das atividades realizadas se encontram nos Anexos 1 e 2 . 
Tabela 1 Atividades realizadas na imersão.

\begin{tabular}{l} 
Aulas no ISCSP \\
\hline GCO - Gestão e Controle Orçamental \\
PO - Política Orçamental \\
GRHAP - Gestão de Recursos Humanos na Administração Pública \\
SPOP - Serviço Partilhado entre Órgãos Públicos \\
DIPP - Desenho e Implementação de Políticas Públicas \\
AQ - Avaliação da Qualidade do ISCSP \\
MPAD - Metodologia de Pesquisa na Administração Pública \\
PPS - Políticas Públicas para Saúde \\
CAPP- Centro de Administração e Políticas Públicas do ISCSP \\
\hline \multicolumn{2}{c}{ Visitas Técnicas } \\
\hline ANQEP - Agência Nacional para Qualificação e Ensino Profissional \\
INA - Direção Geral da Qualificação. Trabalhadores em Funções Públicas \\
CNDH - Comissão Nacional para os Direitos Humanos \\
DGAEP - Direção Geral da Administração e do Emprego Público \\
MNA - Museu Nacional do Azulejo
\end{tabular}

\subsection{DADOS UTILIZADOS}

Ao final das atividades no ISCSP aplicou-se o questionário do Anexo 3 para todos os alunos que participaram da imersão, totalizando 26 questionários inteiramente respondidos.

O questionário conteve perguntas fechadas que mensuraram em uma escala ordinal de cinco pontos o grau de importância da imersão, a sua relação benefício/custo e o grau de dificuldade para a participação dos alunos. Na sequência de cada questão fechada foi incluída uma questão aberta para coletar as sugestões dos alunos em relação às dimensões analisadas. Ao fim do questionário foi deixado um espaço aberto para os alunos expressarem suas considerações gerais sobre a atividade.

Além do rol de perguntas, o questionário incluiu três perguntas fechadas que permitiram segmentar os alunos quanto a sua instituição laboral de origem (Institutos Federais - IF ou Universidade de Brasília - UnB) e quanto ao seu nível de interesse na imersão e no mestrado do PPGP. Essas questões permitiram uma análise desagregada dos resultados.

\subsection{INSTRUMENTAL ANALÍTICO}

Os métodos empregados para analisar as respostas do questionário foram: estatística descritiva e teste não paramétrico de Mann-Whitney para as questões fechadas e análise de conteúdo para as perguntas abertas.

O teste de Mann-Whitney foi utilizado para identificar a existência de diferença significativa entre a percepção dos alunos quanto à contribuição de cada atividade realizada 
na imersão (aulas e visitas técnicas), permitindo apontar o grupo de atividades que foram mais e menos importantes para a sua formação.

Além disso, utilizou-se do mesmo teste para analisar as respostas desagregadas da amostra, verificando a existência de diferença estatisticamente significativa entre a percepção dos alunos da UnB e do IF e dos mais e menos interessados com a imersão e com o PPGP.

\section{RESULTADOS E DISCUSSÕES}

\subsection{CONTRIBUIÇÕES DA IMERSÃO PARA A FORMAÇÃO DOS ALUNOS}

A maioria dos alunos respondeu que a contribuição da imersão em sua formação foi extremamente importante e nenhuma resposta a indicou como pouco ou sem importância (Figura 1).

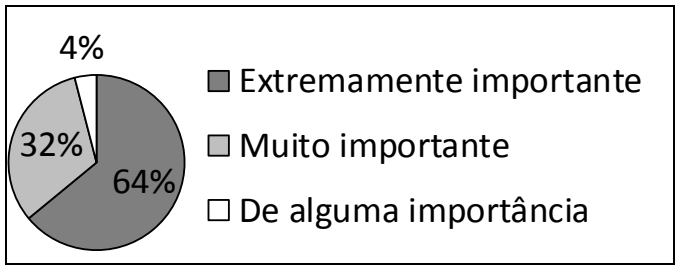

Figura 1 Percepção dos alunos em relação à contribuição da imersão para a sua formação.

A seguir alguns comentários que refletiram a contribuição da imersão para a formação dos alunos:

\footnotetext{
No geral atendeu muito bem as minhas necessidades, pude obter diversos benefícios como: cultura local, economia, administração pública, dentre outros (Aluno 19). Excelente oportunidade para enriquecimento cultural e intelectual (Aluno 10). Achei a imersão muito importante e necessária. Foi uma experiência muito boa e que deve ser repetida aos próximos alunos (Aluno 7).

[...] essa experiência me possibilitou comparações e ideias de mudanças não só para meu setor, como para a instituição como um todo e até para o município em que moro (Aluno 9).

[...] a imersão em Portugal proporcionou ter uma visão ampliada em contexto mundial, sobretudo europeu, das organizações públicas. Com certeza o que aprendemos aqui em Portugal e vivenciamos dentro de suas instalações e sociedade modificou a forma de pensar e as nossas futuras tomadas de decisões (Aluno 1).

Mudou minha percepção de ensino e perspectiva de estudo (Aluno 3).

[...] enriquecedor culturalmente e cientificamente (Aluno 21).
}

Dos 26 alunos investigados apenas dois não apontaram sugestões, corroborando com um grande interesse dos discentes em participar da avaliação e melhoria do programa. As sugestões dos alunos para melhoria da imersão foram difusas, não havendo a predominância 
de nenhum aspecto em particular. A partir do conteúdo das respostas dos alunos pode-se resumir as respostas nas sugestões da Tabela 2 .

Tabela 2 Número de comentários alinhados com as sugestões para ampliação das contribuições da imersão.

\begin{tabular}{cl}
\hline $\begin{array}{c}\text { Número de } \\
\text { comentários }\end{array}$ & \multicolumn{1}{c}{ Sugestão } \\
\hline 5 & Aumento da disponibilidade de disciplinas e possibilidade dos alunos frequentarem \\
4 & aulas conforme suas necessidades \\
3 & Aumento da carga horária de aulas \\
2 & Atividades voltadas à prática, principalmente, em relação às visitas técnicas \\
2 & Maior direcionamento das aulas para a gestão pública \\
2 & Aulas diferentes das cursadas no PPGP ou mais avançadas \\
2 & Aumentibilidade de realizar uma disciplina completa \\
2 & Redução e concentração do período de imersão para uma semana \\
\hline
\end{tabular}

Em linhas gerais, as sugestões dos alunos para a ampliação dos benefícios da imersão para sua formação se referem à disponibilidade de aulas e atividades no ISCSP mais especificas as suas necessidades e visitas mais práticas. A dificuldade no atendimento dessas sugestões deve-se a dificuldades logísticas que foram ampliadas devido ao tamanho da turma que participou da imersão.

Não foi identificada uma contribuição estatisticamente diferenciada entre os eventos no ISCSP (predominantemente aulas) e fora do ISCSP (visitas técnicas), refletindo a importância de ambas as atividades para a imersão. Em uma escala de 0 a 10, a nota média atribuída para as atividades no ISCSP e fora do ISCSP foram de 8,7 e 8,6, respectivamente.

A avaliação individual das atividades por meio do teste não paramétrico de MannWhitney ao nível de 5\% de significância (Tabela 3) possibilitou resumir o conjunto de atividades em três grupos e classificá-las conforme o grau de importância: 1) Extremamente Importantes; 2) Muito Importantes; 3) De Alguma Importância (Tabela 4). 
Tabela 3 Resultados do Teste de Mann-Whitney.

\begin{tabular}{cccccc}
\hline Grupo 1 & Grupo 2 & Grupo 3 & Grupo 4 & Grupo 5 & Grupo 6 \\
\hline GCO & & & & & \\
INA & INA & DGAEP & & \\
DGAEP & DGAEP & PO & & \\
PO & PO & PO & & \\
GRHAP & GRHAP & GRHAP & ANQEP & & \\
ANQEP & ANQEP & ANQEP & SPOP & & \\
& SPOP & SPOP & DIPP & & \\
& & DIPP & AQ & & \\
& AP & MPAP & PPS & \\
& & PPS & PPS & CAPP2 & CAPP1 \\
& & & CAPP1 & MNA & MNA \\
& & & & CNDH \\
\hline
\end{tabular}

Nota: GCO (Gestão e Controle Orçamental); PO (Política Orçamental); GRHAP (Gestão de Recursos Humanos na Administração Pública); SPOP (Serviço Partilhado entre Órgãos Públicos); DIPP (Desenho e Implementação de Políticas Públicas); AQ (Avaliação da Qualidade do ISCSP); MPAP (Metodologia de Pesquisa na Administração Pública); PPS (Políticas Públicas para Saúde); CAPP1 (Estrutura do CAPP); CAPP2 (Exemplos de projetos do CAPP); INA (Direção Geral da Qualificação de Trabalhadores em Funções Públicas); DGAEP (Direção Geral da Administração e do Emprego Público); ANQEP (Agência Nacional para Qualificação e Ensino Profissional); MNA (Museu Nacional do Azulejo); CNDH (Comissão Nacional para os Direitos Humanos).

Tabela 4 Classificação da contribuição das atividades para a formação dos alunos.

\begin{tabular}{cc}
\hline Atividades & Grau de Importância \\
\hline GCO & \\
INA & Extremamente importantes \\
DGAEP & \\
PO & \\
GRHAP & \\
ANQEP & Muito importante \\
SPOP & \\
DIPP & \\
AQ & \\
MPAP & De alguma importância \\
PPS & \\
CAPP2 & \\
CAPP1 &
\end{tabular}

As atividades extremamente importantes estiveram relacionadas às visitas aos órgãos que trabalham diretamente com a administração pública (INA, DGAEP e ANQEP) e as aulas que trataram sobre assuntos ligados à área de orçamento e gestão de Recursos Humanos RH.

Os motivos que podem explicar a maior contribuição das disciplinas na área de orçamento e gestão de RH comparativamente as disciplinas classificadas com um grau de importância imediatamente inferior (Serviço Partilhado, Desenho e Implementação de 
Políticas Públicas, Avaliação da Qualidade do ISCSP, Metodologia de Pesquisa, Políticas Públicas para a Saúde), podem estar relacionados a:

- Melhor didática e grande experiência profissional dos professores que ministraram as disciplinas;

- Aulas que podem estar mais próximas da realidade do aluno. No caso das disciplinas de orçamento por terem abordado questões macroeconômicas que fazem parte do noticiário diário, no caso de gestão de RH por fazer parte do cotidiano da vida profissional dos alunos;

- Aula de gestão de RH no serviço público ter dialogado com a visita técnica no INA, possibilitando uma sinergia entre as atividades. Além disso, o Brasil se encontra em uma situação bem diferenciada de Portugal no que tange a gestão de RH no serviço público, pois o modelo português é mais próximo do que normalmente é feito no setor privado, possivelmente esse aspecto pode ter despertando um maior interesse dos alunos;

- Maior participação nas aulas pelos professores que acompanharam os alunos, facilitando o acompanhamento pela turma, principalmente em relação às aulas de orçamento que faz parte da área de atuação dos dois professores do PPGP presentes;

- Aulas de orçamento e gestão de RH estiveram mais próximas de uma palestra e não de uma aula com necessidade de continuação, possivelmente esse formato facilitou o acompanhamento pelos alunos.

Os possíveis motivos que levaram os alunos a perceberem as atividades CAPP1, MNA

e CNDH como de menor contribuição na sua formação podem estar relacionadas aos seguintes motivos:

- CAPP1 foi uma aula que se ocupou de mostrar o desenho e a estrutura do Centro de Pesquisa da Universidade de Lisboa, provavelmente de pouco interesse pelos alunos, porém de grande interesse dos professores do PPGP e da direção da FUP com vistas a ampliação da cooperação com o ISCSP. Como a aula do CAPP2 se ocupou em exemplificar alguns projetos realizados pelos professores do ISCSP o seu interesse pelos alunos foi maior;

- A visita ao MNA provavelmente foi entendida como um evento cultural e pouco acadêmico, não sendo compreendida a sua importância para a formação dos alunos; 
- Por fim, a baixa contribuição da visita da CNDH na percepção dos alunos pode estar relacionada à especificidade dos assuntos tratados que podem não fazer parte do interesse de muitos alunos, pois se ocupou em mostrar como Portugal lida com o direitos das minorias.

\subsection{CUSTO/BENEFÍCIO DA IMERSÃO PARA INSTITUIÇÃO DE ORIGEM}

A percepção do custo/benefício da imersão obteve resultados similares aos obtidos em relação à contribuição da imersão para a formação dos alunos, ou seja, a maioria dos participantes indicou um custo/benefício extremamente favorável da imersão para a sua instituição laboral. Esses resultados sugerem que o impacto no desempenho profissional adquirido pela imersão compensou com facilidade o investimento feito pela instituição de origem (Figura 2).

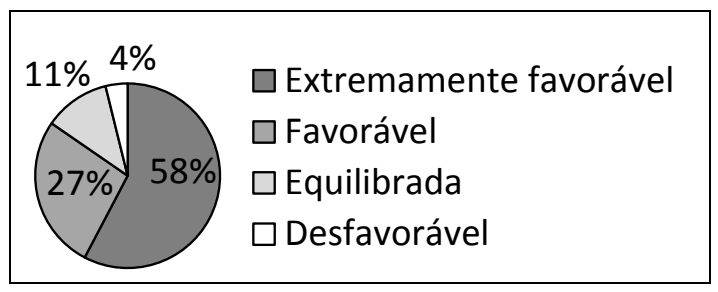

Figura 2 Percepção dos alunos quanto à relação benefício/custo da imersão para a sua instituição.

A maioria dos alunos conseguiram financiamento de suas instituições laborais para as passagens e diárias, ressaltando que os funcionários da UnB obtiveram valores de meia diária e alguns alunos do IF custearam a maior parte da sua imersão com recursos próprios, recebendo apenas as passagens aéreas. Esses motivos reduziram os custos para a sua instituição laboral de origem e contribuíram para a percepção positiva dos alunos na relação benefício/custo da imersão, conforme os seguintes comentários:

\footnotetext{
Acredito que devido à instituição ter disponibilizado recursos abaixo do necessário para realização da imersão, financeiramente ela obteve benefício se relacionado ao custo. Se tomar como foco o conhecimento e vivencia adquiridas nesta imersão, seguramente a instituição teve excelentes benefícios (Aluno 2).

Minha instituição arcou com um valor bastante pequeno, que não cobriu todas as atividades e custos, nesse caso a relação benefício/custo foi bastante grande para a instituição que terá um profissional com maior visão e qualificação com pouco investimento (Aluno 25).
} 
As sugestões para a melhoria da relação benefício/custo da imersão para a instituição de origem foram diversas e muitos comentários fugiram do tema, refletindo a insatisfação dos alunos em relação à burocracia e dificuldade de obterem recursos em suas instituições.

Porém, dois aspectos que podem melhorar a relação benefício/custo da imersão obtiveram um maior consenso entre os participantes. O primeiro, com cinco opiniões, relacionado a questões que podem levar ao melhor preenchimento da grade horária no período de imersão. O segundo, com quatro opiniões, diz respeito a um maior empenho da instituição de origem (UnB e IF) na valorização e aproveitamento dos conhecimentos adquiridos pelos alunos na imersão.

\subsection{DIFICULDADES ENFRENTADAS PELOS ALUNOS PARA A PARTICIPAÇÃO NA IMERSÃO}

Metade dos alunos declararam que foi difícil participar da imersão em Portugal, sete afirmaram que não foi nem difícil e nem fácil e para quatro alunos a participação foi fácil. Nos extremos, um aluno declarou ter sido extremamente difícil e outro extremamente fácil (Figura 3).

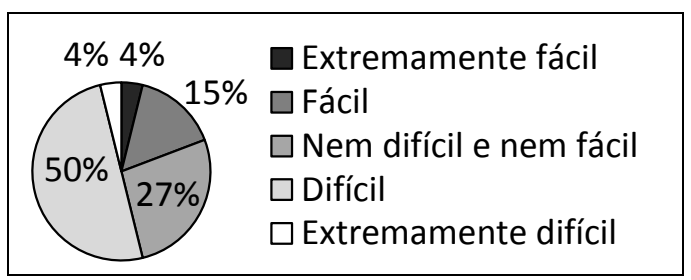

Figura 3 Percepção dos alunos quanto à dificuldade de participar na imersão em Portugal.

Embora a maioria dos alunos tenham declarado que tiveram dificuldades para participar da imersão os empecilhos não foram limitantes, pois dos 32 alunos das duas primeiras turmas apenas seis não fizeram parte do grupo e, dois desses, por motivos compreensíveis de recém-maternidade.

As sugestões dos alunos para facilitar a sua participação estiveram relacionadas, em sua grande maioria, a facilitação na obtenção de recursos. Em geral, as sugestões dos alunos podem ser resumidas em duas dimensões:

1. Maior organização e empenho das instituições (UnB, PPGP e Secretaria de Educação Profissional e Tecnológica - SETEC) para conseguir a liberação de recursos em tempo hábil e em quantidade suficiente, contando com 13 opiniões nessa direção como, por exemplo: 
O pagamento das diárias deveria ter sido integral e em tempo hábil, pois muitos alunos tiveram que pegar ou arrumar algum recurso emprestado (Aluno 7). A própria UnB ter intermediado o processo de diárias e passagens (Aluno 3).

Maior organização da coordenação do PPGP. Mais empenho pessoal em acompanhar a disponibilização de recursos (Aluno 8).

O PPGP poderia ter tido mais rapidez no envio dos documentos relativos à imersão para que os processos fossem abertos mais cedo o que não aconteceu e prejudicou a vinda de alguns colegas (Aluno 24).

Comunicação da SETEC com os institutos federais de forma clara para não haver dúvidas sobre a parceria e padronização das diárias (Aluno 13).

2. Alteração do período da imersão para facilitar a obtenção de recursos com quatro opiniões como, por exemplo:

O período de imersão deve ser entre abril e outubro em função da disponibilidade orçamentária (Aluno 4).

Alteração da data de imersão, pois coincidiu com o início do exercício financeiro sendo este um período difícil para liberação de recursos. Após o $2^{\circ}$ semestre acredito ser um período mais vantajoso financeiramente [...] (Aluno 20).

As dificuldades de ordem financeira enfrentadas pelos alunos foram esperadas. Marrara (2007) apontou que atividades de internacionalização similares a que foi realizada, ou seja, de dentro para fora, exigem pesados investimentos de modo que, no Brasil, sua realização somente tem sido (e continuará sendo) possível graças à atuação das agências federais, como a Capes, o CNPq e algumas agências estaduais de amparo à pesquisa.

\subsection{RESULTADOS DESAGREGADOS POR INSTITUIÇÃO DE ORIGEM E NÍVEL DE INTERESSE COM A IMERSÃO E COM O MESTRADO PROFISSIONAL}

Na percepção dos entrevistados, a contribuição da imersão percebida pelos alunos do IF foi estatisticamente superior à dos alunos da UnB (Tabela 5). Observando as sugestões nesse aspecto, em desagregado por instituição laboral de origem, percebeu-se uma maior cobrança por atividades práticas aplicadas a gestão pública pelos alunos da UnB, provavelmente essa tenha sido uma questão importante para explicar a diferença desses resultados. 
Tabela 5 Mediana das respostas desagregadas pela instituição de origem dos servidores e pelo nível de interesse com a imersão e com o mestrado profissional.

\begin{tabular}{|c|c|c|c|c|c|c|}
\hline & \multicolumn{2}{|c|}{$\begin{array}{l}\text { Instituição } \\
\text { Origem } \\
\text { Servidores }\end{array}$} & \multicolumn{2}{|c|}{$\begin{array}{l}\text { Nível de Interesse com } \\
\text { a Imersão }\end{array}$} & \multicolumn{2}{|c|}{$\begin{array}{l}\text { Nível de Interesse com o } \\
\text { Mestrado Profissional }\end{array}$} \\
\hline & IF & UnB & $\begin{array}{l}10 \% \text { com } \\
\text { maior } \\
\text { interesse }\end{array}$ & $\begin{array}{l}\text { Alunos de } \\
\text { menor } \\
\text { interesse }\end{array}$ & $\begin{array}{l}10 \% \text { com } \\
\text { maior } \\
\text { interesse }\end{array}$ & $\begin{array}{l}\text { Alunos de } \\
\text { menor } \\
\text { interesse }\end{array}$ \\
\hline $\begin{array}{l}\text { Contribuição para } \\
\text { formação }\end{array}$ & $5^{1}$ & $4^{1}$ & $5^{\text {ns }}$ & $5^{\mathrm{ns}}$ & $5^{\text {ns }}$ & $5^{\text {ns }}$ \\
\hline $\begin{array}{l}\text { Benefício/Custo } \\
\text { para Instituição }\end{array}$ & $5^{\mathrm{ns}}$ & $5^{\text {ns }}$ & $5^{\text {ns }}$ & $4^{\mathrm{ns}}$ & $5^{2}$ & $4^{2}$ \\
\hline $\begin{array}{l}\text { Dificuldades para } \\
\text { participação }\end{array}$ & $3^{1}$ & $2^{1}$ & $2^{\text {ns }}$ & $3^{\text {ns }}$ & $2^{\mathrm{ns}}$ & $3^{\text {ns }}$ \\
\hline
\end{tabular}

Nota: ${ }^{1}$ Diferença significativa ao nível de $5 \% ;{ }^{2}$ Diferença significativa ao nível de $10 \%$.

Enquanto 33\% das sugestões dos alunos da UnB para a ampliação da contribuição da imersão em sua formação abordaram a importância de atividades práticas o mesmo percentual para os alunos do IF foi de apenas $7 \%$.

O grau de dificuldade para participar na imersão percebida pelos alunos da UnB foi estatisticamente superior ao dos alunos do IF. A mediana das respostas para os alunos da UnB na escala de cinco pontos se localizou no atributo "Difícil", para os alunos do IF a mesma medida foi classificada como "Nem difícil e nem fácil".

Analisando em desagregado por instituição de origem, as respostas das questões abertas referente às sugestões que poderiam facilitar a participação na imersão, ficou claro uma posição mais crítica dos alunos da UnB em relação ao empenho das instituições (UnB e PPGP) para obtenção e liberação de recursos em tempo hábil e em quantidade suficiente para a sua imersão. Enquanto metade dos alunos da UnB abordou essa questão para o IF esse percentual foi de $36 \%$ das respostas.

Por fim, a relação benefício/custo para os alunos com maior grau de interesse no mestrado profissional foi superior quando comparado com aqueles de menor grau de interesse. Esse resultado foi esperado, pois o empenho do aluno é fundamental para o melhor aproveitamento de qualquer programa de imersão.

Interessa destacar que, diferentemente dos alunos de menor interesse, as sugestões dos 10\% mais interessados com o PPGP se concentraram em questões voltadas para ampliar os benefícios da imersão como, por exemplo: possibilidade de terem mais aulas, maior empenho das instituições laborais de origem no aproveitamento dos conhecimentos adquiridos, entre outras. Para os alunos de menor interesse, a proporção de respostas que utilizou esse espaço se 
concentrou sem sugestões voltadas para redução dos custos da imersão, seja pela redução do período de imersão ou pela disponibilização de alojamento pela instituição de origem, bem como, também atribuíram um maior custo da imersão devido a problemas de organização.

\subsection{CONSIDERAÇÕES GERAIS DOS ALUNOS}

Em geral, o espaço aberto do questionário para os alunos emitirem quaisquer considerações foi utilizado para agradecimentos e elogios à organização da imersão, bem como, se destacou uma incompreensão em relação à participação da vice-diretora da FUP em Portugal.

A maioria dos alunos (57\% das respostas) expressou alguma forma de elogio ou agradecimento à imersão, destacando os seguintes comentários:

\footnotetext{
Parabéns a toda a turma pela disponibilidade em participar da imersão e com toda a equipe de docentes e técnicos envolvidos (Aluno 2).

Só tenho a agradecer a oportunidade que a UnB e IF me proporcionou, agradeço aos professores envolvidos nesta difícil logística da imersão e acredito nestas iniciativas como crescimento profissional e pessoal (Aluno 10).

Parabenizo a coordenação e professores que nos acompanhou, a programação foi muito boa, a recepção, organização foram perfeitas [...] (Aluno 9).

Os professores que acompanharam portaram-se com muito profissionalismo e dedicação. Parabéns ao programa. Sou muito agradecido (Aluno 8).
}

Em relação à participação da vice-diretora, oito respostas ou $30 \%$ dos alunos manifestaram alguma forma de incompreensão ou insatisfação com a sua presença, podendo citar:

Ficou nítido a insatisfação com a vinda não explicada da vice-diretora. Sabemos que é necessária articulação na parceria, mas a impressão que passou é que ela veio a passeio [...]. Vale a pena avaliar o custo benefício da vinda dela para a instituição e o programa (Aluno 25).

[...] só não entendi a participação da vice-diretora que não foi aos eventos (Aluno 9). Penso que a vinda da vice-diretora não foi necessária o que só onerou os cofres públicos, tendo em vista que ela não teve participação nenhuma no nosso PPGP (Aluno 24).

A participação da vice-diretora não foi bem compreendida pelos alunos, indicando a necessidade de esclarecimento da importância institucional da sua presença na formalização e expansão do termo de cooperação com a Universidade de Lisboa, bem como, o estabelecimento de uma agenda compatível com suas atividades no país visitado.

Nesse aspecto se destaca os resultados do estudo de Duarte et al. (2009). Os autores apontaram que um dos principais desafios do processo de internacionalização das IES reside na institucionalização do processo que, em geral, é informal o que tem levado a uma 
dependência da instituição a ações isoladas dos docentes. Em muitos casos, os benefícios para instituição de ações isoladas dos docentes são questionados o que eleva a importância do envolvimento da direção no processo. Algumas críticas dessas ações decorrem, por exemplo, quando docentes utilizam recurso público nacional para realizar cursos internacionais de capacitação voltados para o seu interesse pessoal ou quando utilizam desse recurso para realizar e publicar pesquisas voltadas para resolução de problemas de outros países.

\section{CONCLUSÕES}

A partir da percepção dos alunos, os resultados ensejam as seguintes conclusões. Em primeiro lugar, a imersão internacional em Portugal foi extremamente relevante para a formação dos alunos em Gestão Pública, valendo o esforço do PPGP na aproximação com o ISCSP e continuidade da iniciativa para novas turmas. Tal conclusão aponta para o complexo paradigma que trata do processo de internacionalização do conhecimento, que é um dos elementos visíveis do internacionalismo da educação superior (ALTBACH, 2009).

Em segundo lugar, o investimento financeiro desta aproximação foi recompensado pelo impacto no ambiente de trabalho decorrente da formação de seus servidores. Para melhorar a relação de benefício/custo da imersão a organização deve trabalhar na direção de tornar as atividades no País de destino mais práticas, além disso, é importante que as instituições laborais de origem valorizem o conhecimento adquirido por seus servidores.

Por outro lado, a internacionalização não é um fato definitivo, nem apenas um marco, mas um processo (MARTINS, 2010). Desta forma, dificuldades são apresentados, pois a obtenção de recursos públicos para a participação dos estudantes esteve presente. Talvez por serem servidores públicos, é notório uma cultura entre os alunos de que o Estado deve custear toda a sua formação e, além disso, de que a burocracia para liberação do recurso público para atividade de interesse do aluno deve ser de responsabilidade do programa de pós-graduação. Como sugestão, o PPGP, por meio de todos os seus professores, em suas disciplinas, deve atuar para a mudança dessa cultura paternalista.

Um aspecto que deve ser trabalhado e melhor comunicado para os alunos é o papel de membros que representem a direção da UnB no acompanhamento da imersão. Os alunos devem ser comunicados que o papel da direção é institucional e que, necessariamente, não precisam acompanhar todas as atividades de cunho pedagógico. 
Em função desta avaliação refletir apenas a percepção dos alunos torna-se limitada, não minimizando a sua importância, apenas deve ter seus resultados interpretados de forma cautelosa dado que o processo como um todo é recente. Destaca-se aqui que o primeiro passo em direção à marca das relações entre as universidades foi dado, no entanto, é preciso lembrar que de modo geral essa relação está em fase de construção.

\section{REFERÊNCIAS BIBLIOGRÁFICAS}

ALTBACH, P. G. Educación superior comparada: el conocimiento, la universidad y el desarrollo. $1^{\text {a }}$ ed. Buenos Aires: Universidad de Palermo - UP, 2009.

BARTELL, M. Internationalization of universities: A university culture-based framework. Higher Education. Manitoba, Winnipeg, 2003, p. 37-52. In: MOROSINI, M. C. Estado do conhecimento sobre internacionalização da educação superior. Educar, n. 28, p. 107-124, 2006.

DAVIES, J. L. A revolution in teaching and learning in higher education: the challenges and implications for the relatively traditional university. Higher Education in Europe, v. 26, n. 4, p. 501-514, 2001.

DENMAN, B. D. Higher Education Management and Policy. Higher Education Management and Policy, v. 16, n. 1, p. 65-82, 2004.

DUARTE, R. G.; CASTRO, J. M. de; PEREIRA, A. C. C.; CRUZ, A. L. A. O papel dos relacionamentos interpessoais no processo de internacionalização das Instituições de Ensino Superior (IES). Anais do Encontro da Associação Nacional de Pós-graduação e Pesquisa em Administração, São Paulo, SP, Brasil, 2009.

KNIGHT, J. Internationalization: elements and checkpoints. CBIE Research, n. 7, 1994.

KNIGHT, J. Internationalization remodeled definition, approaches and rationales. Journal of Studies in International Education, v. 8, n. 1, p. 5-31, 2004.

KOK, J. A. The internationalization of universities through the management of their intelectual capital. In: Anais da 6th International Conference da Faculty of Management Koper. Eslovenia, 24 a 26 de novembro de 2005.

LAUS, S. P. A internacionalização da educação superior: um estudo de caso da Universidade Federal de Santa Catarina. Tese (Doutorado). Universidade Federal da Bahia, Escola de Administração, 2012.

MARRARA, T. Internacionalização da Pós-Graduação: objetivos, formas e avaliação. Revista Brasileira de Pós-Graduação, Brasília, v. 4, n. 8, p. 245-262, 2007.

MARTINS, J. R. A Unila e a integração Sul-Americana: um projeto de liderança regional brasileira, 2010. Disponível em: http://www.administradores.com.br/artigos/administracaoe- 
negocios/a-unila-e-a-integracao-sul-americana-um-projeto-de-lideranca-regional-brasileira /46972/>. Acesso em: 04 ago. 2015.

MOROSINI, M. C. Estado do conhecimento sobre internacionalização da educação superior. Educar, n. 28, p. 107-124, 2006.

MOK, K. H. Questing for internationalization in East Asia: critical reflexions. In: Anais do Simpósio Internacional da Universidade de Osaka, 13 a 14 de janeiro de 2006.

RUDZKI, R. E. J. The strategic management of internationalization: towards a model of theory and practice. Thesis, School of Education, University of Newcastle upon Tyne, United Kingdom, 1998.

SEBASTIÁN, J. Cooperación e Internacionalización de las Universidades. Buenos Aires: Biblos, 2004. 
Anexo 1 Agenda das atividades realizadas

\begin{tabular}{|c|c|c|c|c|}
\hline \multicolumn{5}{|c|}{ Semana 1} \\
\hline Horário & $23 /$ fev & $24 /$ fev & $25 /$ fev & $26 /$ fev \\
\hline $10 \mathrm{~h} 00$ & $\begin{array}{c}\text { Recepção } \\
\text { Institucional no } \\
\text { ISCSP } \\
\end{array}$ & MNA - Museu & $\begin{array}{c}\text { ANQEP - Agência } \\
\text { Nacional para }\end{array}$ & $\begin{array}{l}\text { CAPP - Centro de } \\
\text { Administração e }\end{array}$ \\
\hline $\begin{array}{l}11 \mathrm{~h} 00 \\
13 \mathrm{~h} 00\end{array}$ & $\begin{array}{c}\text { Visita às instalações } \\
\text { Pedro de Abreu }\end{array}$ & & $\begin{array}{l}\text { Qualıfıcaçao e } \\
\text { Ensino Profissional }\end{array}$ & Políticas Públicas \\
\hline $13 \mathrm{~h} 00$ & \multicolumn{4}{|c|}{ Almoço } \\
\hline $\begin{array}{l}14 \mathrm{~h} 00 \\
16 \mathrm{~h} 00\end{array}$ & & & & \\
\hline $\begin{array}{l}16 \mathrm{~h} 00 \\
18 \mathrm{~h} 00\end{array}$ & & & $\begin{array}{c}\text { Gestão e Controle } \\
\text { Orçamental. } \\
\text { Prof. Albano } \\
\text { Santos }\end{array}$ & $\begin{array}{c}\text { Serviço Partilhado. } \\
\text { Prof. Helena } \\
\text { Monteiro }\end{array}$ \\
\hline $\begin{array}{l}\text { 20h } 00 \\
22 \mathrm{~h} 00\end{array}$ & $\begin{array}{l}\text { Desenho e } \\
\text { Implementação de } \\
\text { Políticas Públicas. } \\
\text { Prof. Marta Rocha }\end{array}$ & $\begin{array}{c}\text { Metodologia de } \\
\text { Pesquisa na } \\
\text { Administração Pública. } \\
\text { Prof. Fernanda } \\
\text { Nogueira }\end{array}$ & & \\
\hline
\end{tabular}

\begin{tabular}{|c|c|c|c|c|}
\hline \multicolumn{5}{|c|}{ Semana 2} \\
\hline Horário & $23 / \mathrm{fev}$ & $24 / \mathrm{fev}$ & $25 / \mathrm{fev}$ & $26 / \mathrm{fev}$ \\
\hline $\begin{array}{l}10 \mathrm{~h} 00 \\
13 \mathrm{~h} 00\end{array}$ & $\begin{array}{c}\text { INA - Direção } \\
\text { Geral da } \\
\text { Qualificação. } \\
\text { Trabalhadores em } \\
\text { Funções Públicas }\end{array}$ & $\begin{array}{c}\text { CNDH - Comissão } \\
\text { Nacional para os Direitos } \\
\text { Humanos }\end{array}$ & $\begin{array}{c}\text { DGAEP - Direção } \\
\text { Geral da } \\
\text { Administração e } \\
\text { do Emprego } \\
\text { Publico }\end{array}$ & $\begin{array}{l}\text { CAPP - Centro de } \\
\text { Administração e } \\
\text { Políticas Públicas }\end{array}$ \\
\hline $13 \mathrm{~h} 00$ & \multicolumn{4}{|c|}{ Almoço } \\
\hline $\begin{array}{l}14 \mathrm{~h} 00 \\
16 \mathrm{~h} 00\end{array}$ & & & & \\
\hline $\begin{array}{l}16 \mathrm{~h} 00 \\
18 \mathrm{~h} 00\end{array}$ & & $\begin{array}{l}\text { Avaliação da Qualidade } \\
\text { do ISCSP. Prof. Silvia } \\
\text { Vicente }\end{array}$ & $\begin{array}{c}\text { Política } \\
\text { Orçamental. } \\
\text { Prof. Pedro } \\
\text { Rodrigues }\end{array}$ & $\begin{array}{l}\text { Gestão de Recursos } \\
\text { Humanos na } \\
\text { Administração } \\
\text { Pública. Prof. José } \\
\text { Nascimento }\end{array}$ \\
\hline $\begin{array}{l}20 \mathrm{~h} 00 \\
22 \mathrm{~h} 00\end{array}$ & $\begin{array}{l}\text { Políticas públicas } \\
\text { para a Saúde. } \\
\text { Prof. Margarida }\end{array}$ & $\begin{array}{l}\text { Metodologia de Pesquisa } \\
\text { na Administração Pública. } \\
\text { Prof. Fernanda Nogueira }\end{array}$ & & \\
\hline
\end{tabular}




\section{IMERSÃO INTERNACIONAL: UMA AVALIAÇÃO DE INTERCAMBISTAS DE MESTRADO PROFISSIONAL \\ DOI: http://dx.doi.org/10.5007/1983-4535.2017v10n1p280}

Anexo 2 ISCSP e registro de algumas atividades realizadas

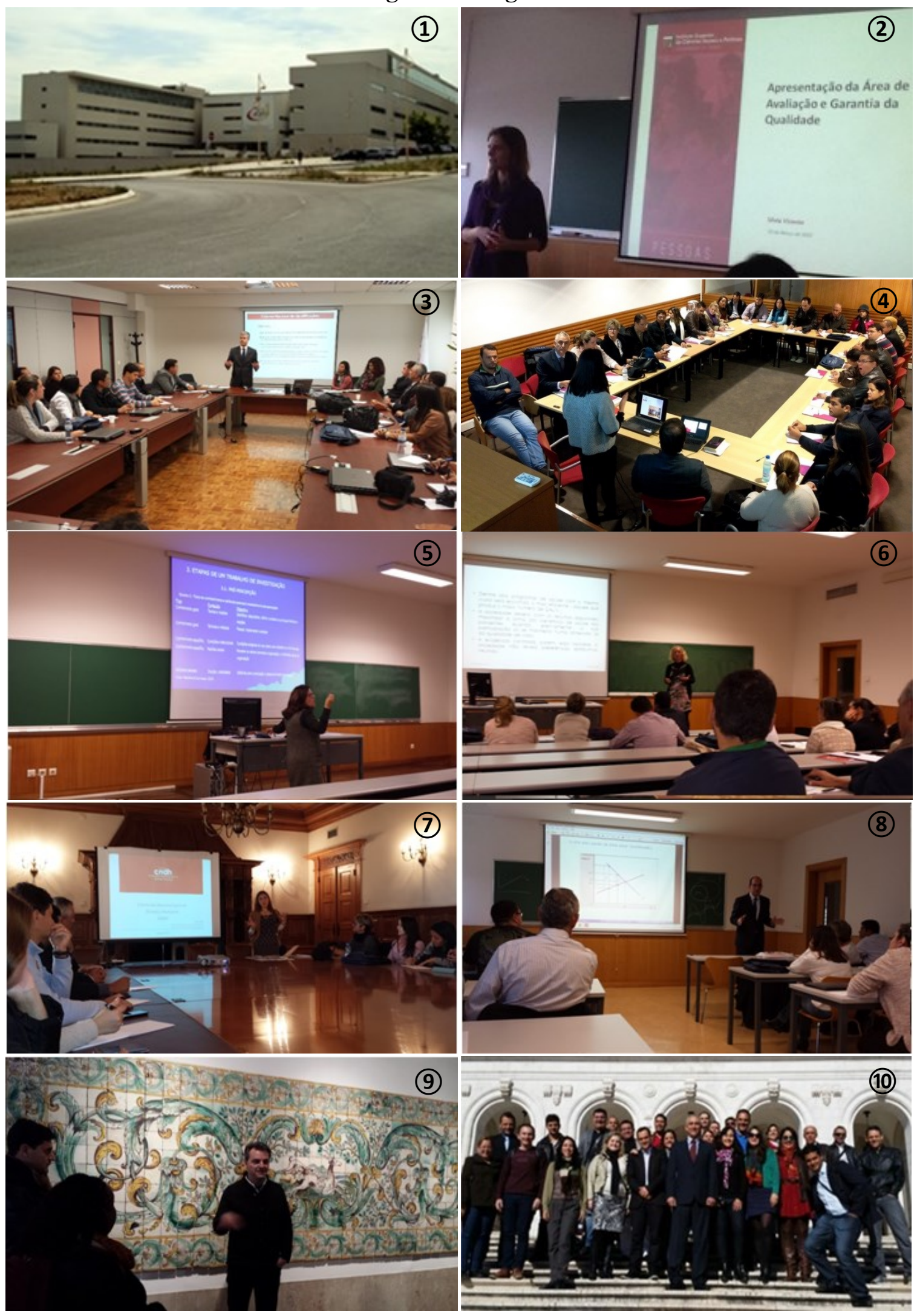

Nota: (1) ISCSP, (2) Avaliação da Qualidade no ISCSP, (3) ANQEP, (4) INA, (5) Metodologia de Pesquisa, (6) Política Pública para Saúde, (7) CNDH, (8) Política Orçamental, (9) MNA, (10) Parlamento Português. 


\section{Anexo 3 Questionário aplicado}

\section{Avaliação da Efetividade da Imersão em Portugal - PPGP}

1) Em geral, qual a contribuição da Imersão em Portugal para sua formação no PPGP.

( ) Totalmente sem importância

( ) Pouco importante

( ) De alguma importância

( ) Muito importante

( ) Extremamente importante

2) Por favor, indique sugestões que poderiam ter ampliado a contribuição da imersão em Portugal para sua formação.

3) Atribua uma nota de 0 a 10 para as atividades no ISCSP e fora do ISCPS.

( ) Atividades no ISCSP

( ) Atividades fora do ISCPS

4) Indique a contribuição individual de cada atividade para a sua formação conforme a seguinte escala:

(1) Totalmente sem importância

2 Pouco importante

3 De alguma importância

$(4$ Muito importante

(5) Extremamente importante

\begin{tabular}{|c|c|c|c|c|c|}
\hline \multirow{2}{*}{ Atividades } & \multicolumn{5}{|c|}{ Escala } \\
\hline & (1) & 2 & 3 & 4 & 5 \\
\hline \multicolumn{6}{|l|}{ MNA - Museu Nacional do Azulejo } \\
\hline \multicolumn{6}{|c|}{ ANQEP - Agencia Nacional para Qualificação e o Ensino Profissional } \\
\hline \multicolumn{6}{|c|}{$\begin{array}{l}\text { INA - Direção Geral da Qualificação. Trabalhadores em Funções } \\
\text { Publicas }\end{array}$} \\
\hline \multicolumn{6}{|c|}{ CNDH-Comissão Nacional para os Direitos Humanos } \\
\hline \multicolumn{6}{|c|}{ DGAEP - Direção Geral da Administração e do Emprego Publico } \\
\hline \multicolumn{6}{|c|}{ Aula: Desenho e Implementação de Políticas Públicas } \\
\hline \multicolumn{6}{|c|}{ Aula: Metodologias de Pesquisa na Administração Pública } \\
\hline \multicolumn{6}{|l|}{ Aula: Políticas públicas para saúde } \\
\hline \multicolumn{6}{|l|}{ Aula: Gestão e Controle Orçamental } \\
\hline \multicolumn{6}{|l|}{ Aula: Serviço Partilhado } \\
\hline \multicolumn{6}{|l|}{ Aula: Política Orçamental } \\
\hline \multicolumn{6}{|c|}{ Aula: Avaliação da Qualidade do ISCSP } \\
\hline \multicolumn{6}{|c|}{ Aula: Gestão de Recursos Humanos na Administração Pública } \\
\hline \multicolumn{6}{|l|}{ CAPP 1. Introdução } \\
\hline CAPP 2. Apresentação de projetos & & & & & \\
\hline
\end{tabular}


5) Qual sua instituição de origem?

( ) Instituto Federal (IF) ( ) Universidade de Brasília (UnB)

6) Em sua opinião, a relação custo/benefício da sua imersão em Portugal para sua instituição de origem foi:

( ) Extremamente Desfavorável ( ) Desfavorável ( ) Equilibrada ( ) Favorável

( ) Extremamente Favorável

7) Por favor, indique sugestões que poderiam melhorar a relação custo/benefício da sua imersão para a sua instituição?

8) Qual o grau de dificuldade enfrentado para atender à exigência do curso e participar da Imersão em Portugal?

( ) Extremamente difícil ( ) Difícil ( ) Nem Difícil Nem Fácil ( ) Fácil

( ) Extremamente fácil

9) Por favor, indique sugestões que poderiam ter facilitado a sua participação na imersão em Portugal.

10) Comparado aos seus colegas de classe o seu NÍVEL DE INTERESSE com a imersão em Portugal estava:

( ) entre os $10 \%$ dos alunos com maior grau de interesse da turma

( ) entre os $20 \%$ dos alunos com maior grau de interesse da turma

( ) entre os $50 \%$ dos alunos com maior grau de interesse da turma

( ) entre os alunos de menor interesse da turma.

( ) Não sabe

11) Comparado aos seus colegas de classe o seu NÍVEL DE INTERESSE com a obtenção de conhecimento no Mestrado Profissional está:

( ) entre os $10 \%$ dos alunos com maior grau de interesse da turma

( ) entre os $20 \%$ dos alunos com maior grau de interesse da turma

( ) entre os 50\% dos alunos com maior grau de interesse da turma

( ) entre os alunos de menor interesse da turma.

( ) Não sabe

12) Espaço aberto para considerações sobre a imersão em Portugal que não foram abordados em questões anteriores. 